\title{
VARIATIONS DANS LES TAUX DE LIPIDES PLASMATIQUES EN FONCTION de L'INfeCtion À Plasmodium Falciparum À SÃo TOMÉ
}

\author{
BAPTISTA J.L.*, VERVOORT T.*, VAN DER STUYFT P.*, WÉRY M.*
}

Summary : Lipid plasma levels and PLASMOdIU FalCiPARUM INFECTION IN SÁO TOMÉ.

Changes in lipid plasma levels during malaria attacks have been proposed for use in diagnosis or to assess the severity of the disease. In order to analyse the plasma levels of cholesterol, triglycerides, HDL-C et LDL-C, we compared, in an endemic area (São Tomé island), two groups of patients children infected with Plasmodium falciparum (simple malaria attack and cerebral malaria) with a control group of asymptomatic children. No correlation between lipid plasma levels and disease severity was found. Correlations between lipids and parasitemia or anemia were analysed. The mechanism of plasma lipid changes during attack are discussed

KEY WORDS : malaria, Plasmodium falciparum, cholesterol, triglycerides, HDL, $L D L$, anemia.

\section{Résumé :}

Les variations des niveaux des lipides plasmatiques pendant l'accès ont suggéré l'utilisation de leur dosage comme test diagnostique ou comme indicateur de la sévérité de la maladie. Dans le but d'évaluer le changement des cholestérol total (CT), triglycérides (TG), HDL-cholestérol (HDL-C) et LDL-cholestérol pendant l'accès palustre en région endémique (île de São Tomé), on a sélectionné deux groupes d'enfants infectés par Plasmodium falciparum (accès simple et accès pernicieux) et un groupe contrôle. On n'a pas trouvé de corrélation entre concentration de lipides et sévérité de la maladie, mais pour les TG et HDL-C les différences ont été significatives entre malades et non-malades. Corrélations entre lipides, densité parasitaire et degré d'anémie sont analysées, par rapport aux groupes étudiés. Les mécanismes de la dyslipidémie dans l'accès palustre restent discutés, faisant intervenir probablement plusieurs facteurs.

MOTS CLÉS : paludisme, Plasmodium falciparum, cholestérol, triglycérides, HDL, LDL, anémie.

variés (Maurois et al., 1980) : l'infection rapidement mortelle à $P$. vinckei n'induit pas de dyslipoprotéinémie, l'infection non mortelle à $P$. chabaudi induit une augmentation des triglycérides (TG) et une diminution des lipoproteines de haute densité (HDL-C) et l'infection fatale à $P$. berghei n'entraîne que de faibles altérations. Holz (1977) avait déjà souligné des discordances entre les diverses infections.

L'apparition de dyslipoprotéinémies a aussi été observée à répétition chez l'homme impaludé par P. vivax (Hartmann \& Schneider, 1963; Lambrecht et al., 1978, Maurois et al., 1979) ou P. falciparum (Djoumessi, 1989; Agbedana et al., 1990; CuisinierRayal et al., 1990; Mohanty et al., 1992; Das et al., 1993; Davis et al. 1993a).

Nilsson-Ehle \& Nilsson-Ehle (1990) ont suggéré que la réduction des HDL-C pouvait avoir une utilité dans le diagnostic du paludisme tandis que Mohanty et al. (1992) l'a proposé comme indicateur de la sévérité de la maladie. En biologie clinique, l'évaluation des troubles lipidiques (Rifai \& Warnick, 1991; Sandstad et al., 1992) est basée sur le dosage du cholestérol (CT), des TG, des HDL-C et le calcul des concentrations de lipoprotéines de basse densité (LDL-C). C'est ce que nous avons utilisé pour étudier les niveaux de lipides en région endémique, chez des enfants infectés

\footnotetext{
* Institut de Médecine Tropicale Prince Leopold, Nationalestraat 155, B-2000 Antwerpen, Belgique.

Correspondance: J.L. Baptista.

Fax : (32.3) 24.76.362. - Tel. : (32.3) 24.76.35.
} 
par $P$. falciparum et évaluer des différences en fonction de la sévérité du paludisme.

\section{PATIENTS ET METHODES}

\section{LOCALISATION DE L'ÉTUDE}

L 'étude a été effectuée dans le principal centre de santé du District de Lobata (ville de Guadalupe), sur l'île de São Tomé (République Démocratique de São Tomé et Príncipe), région de mésoendémicité palustre, située à $280 \mathrm{~km}$ au large des côtes gabonaises.

\section{Population ÉTudiéE}

Parmi les enfants âgés de 4 à 14 ans qui arrivaient au centre de santé, on a sélectionné deux groupes de malades avec paludisme à $P$. falciparum suivant les critères de définition d'accès palustre de l'Organisation Mondiale de la Santé (Warrel et al., 1990) :

- Accès simple : enfant fébrile, sans altération de l'état de conscience, avec signes et symptômes évocateurs de paludisme et parasitémie périphérique à $P$. falciparum. La sélection a été faite en choisissant un enfant sur cinq parmi ceux qui se présentaient avec les critères décrits à un jour de la semaine déterminé (le mercredi, pour des raisons de convenance à l'hôpital).

- Accès pernicieux : enfant fébrile, comateux, dont l'état de conscience variable, classé selon le score de Glasgow (Barkin \& Rosen, 1990) ne pouvait pas être rapporté à une autre cause qu'à un accès palustre à $P$. falciparum. Tous les enfants avec accès pernicieux ont été sélectionnés.

L'exclusion de maladies autres que le paludisme a été basée sur l'absence établie d'encéphalopathie virale sur l'île, l'observation clinique, la biochimie du sang, l'examen des urines et du liquide céphalo-rachidien, la radiographie du thorax et la séronégativité pour VIH I (test ELISA-Welcome), VIH II (test ELISA-Quilaban) et HBs Ag (test ELISA - ABBOTT). La recherche de la prise de 4-amino-quinoléines sur les urines (Bruce-Chwatt et al. 1986) et le test de falciformation des érythrocytes (Lévy-Lambert, 1973) devaient être négatifs. Une ponction lombaire a été faite à tous les malades au début de l'hospitalisation; les enfants avec liquide céphalo-rachidien opalescent ou blanc-grisâtre et/ou avec plus de 10 leucocytes par/ul, ont été exclus. Le groupe contrôle ou asymptomatique a été défini comme un enfant considéré en bonne santé, sans pathologie visible, du même groupe d'âge. Le statut de bien portant était confirmé par la négativité d'un examen clinique soigneux, la recherche des hémato- zoaires dans le sang périphérique et tous les examens décrits ci-dessus pour les malades. Ces enfants ont été sélectionnés au hasard parmi ceux qui arrivaient au centre de santé sans autre raison que d'accompagner leur mère ou un autre membre de la famille.

\section{TRAITEMENT DES MALADES}

Jusqu'au moment où la voie orale devenait possible, tous les malades ont été hospitalisés et traités par voie parentérale au dichlorhydrate de quinine (dose de charge de $10 \mathrm{mg}$ de $\mathrm{sel} / \mathrm{kg} / 4$ heures, dans une perfusion d'un soluté physiologique contenant $5 \%$ de dextrose, suivie d'une dose de $25 \mathrm{mg}$ de $\mathrm{sel} / \mathrm{kg} /$ jour $\times 7$ jours).

\section{PARAMÈTRES BIOLOGIQUES}

Le diagnostic de paludisme à $P$. falciparum était posé par l'examen microscopique après coloration au Giemsa du frottis fixé au méthanol et de la goutte épaisse. La densité parasitaire a été estimée en comptant les formes asexués pour 200 leucocytes et exprimée en nombre de parasites/ $\mu l$ de sang (Wéry, 1991).

Une prise de sang sur EDTA a été faite avant le début du traitement. Après centrifugation, le plasma a été stocké à $-20^{\circ} \mathrm{C}$ et envoyé à l'Institut de Médecine Tropicale d'Anvers (IMT). Les frottis et gouttes épaisses ont aussi transférés à Anvers pour réexamen, en particulier pour le contrôle des densités parasitaires.

Les dosages de cholestérol total (CT), de triglycérides (TG) et de lipides de haute densité (HDL-C) ont été réalisés dans les laboratoires de l'IMT sur le plasma des malades conservé à $-20^{\circ} \mathrm{C}$ (Kodak Ektachem Analyser 70-XR) en utilisant les méthodes enzymatiques décrites par Allain et al., 1974 pour le CT et par Spayd et al., 1978 pour les TG. Les taux de lipides de basse densité (LDL-C) (Rifai \& Warnick, 1991) ont été calculés à l'aide de la formule de Friedewald et al., 1972 : [LDL$\mathrm{C}=\mathrm{CT}-\mathrm{HDL}-\mathrm{C}-(\mathrm{TG} / 5)]$. Les valeurs de référence pour tous les paramètres mesurés ont été choisies pour le groupe d'âge de 8 ans (Samuel Meites, 1988), à l'exception des lipides, pour lesquelles nous nous sommes basés dans le même groupe d'âge sur les tables de Rifai \& Warnick (1991) pour la race noire.

\section{Tests STATISTIQUeS}

Les variables continues ont été représentées par la médiane et les quartiles 1 et 3 . Les différences entre les groupes sont evaluées avec le test de KruskalWallis. Les associations entre deux variables continues ont été explorées avec le coefficient de corrélation de Pearson $(r)$. Les différences entre pourcentages ont été testées avec le test de Fisher bilatéral $(\alpha<5 \%$ a été utilisé comme seuil de signification). 


\section{RESULTATS}

D es 88 enfants étudiés, deux ont été retirés de l'étude, à cause de la confirmation a posteriori d'une positivité pour l'HBs Ag. Des 86 enfants restants, 44 avaient un accès simple [17 filles et 29 garçons, âge médian 9,5 ans $\left(q_{1}=7,0 ; q_{3}=13,0\right)$ ], 12 un accès pernicieux $[7$ filles et 5 garçons, âge médian 5,3 ans $\left(q_{1}=4,5 ; q_{3}=5,5\right)$ ] et 30 appartiennent au groupe contrôle [16 filles et 14 garçons, âge médian 9,5 ans $\left.\left(q_{1}=7,0 ; q_{3}=13,0\right)\right]$.

Les valeurs des paramètres biologiques étudiés en fonction de la sévérité de la maladie sont résumés au tableau I.

La sévérité de la maladie est positivement associée a la densité parasitaire et négativement au taux d'hémoglobine. Les taux médians d'hémoglobine sont de 9,4 $\mathrm{g} / \mathrm{dl}$ chez les enfants du groupe contrôle, de $7,8 \mathrm{~g} / \mathrm{dl}$ dans les accès simples et de $5,6 \mathrm{~g} / \mathrm{dl}$ dans les accès pernicieux. Les différences entre groupes sont significatives. Le taux médian de CT est plus bas chez les malades que dans le groupe contrôle (fig. 1). Cette différence est significative pour les accès simples, mais elle est sans rapport avec la sévérité de la maladie (tableau I). Le taux de CT n'est pas corrélé avec le taux d'hémoglobine et la densité parasitaire. En ce qui concerne les taux de TG dans notre série, les valeurs du groupe contrôle se situent dans la fourchette des valeurs normales, tandis que $45,5 \%$ des accès simple et 50,0\% des accès pernicieux présentent un taux augmenté au dessus de $140 \mathrm{mg} / \mathrm{dl}$. Les valeurs augment significativement $(p<0,001)$ entre contrôles et malades (tableau I) et non significativement entre formes simple et sévère de la maladie (fig. 1). Il n'y a pas de corrélation entre le taux des TG et le degré de parasitémie $(r=-0,17)$. Des corrélations faibles mais cependant significatives avec le taux d'hémoglobine $(r=-0,37$; $p<0,05)$ ont été trouvées.

En ce qui concerne l'HDL-C, les taux sont diminués chez $70,0 \%$ des sujets du groupe contrôle, 93,2 \% des accès simples et $91,7 \%$ des accès pernicieux. La diminution des HDL-C présente une corrélation significative avec le fait d'être malade $(p<0,001)$ mais pas avec la gravité de la maladie. Comme test diagnostique de présomption du paludisme, le dosage des HDL-C a une sensibilité de 95,0\% mais il souffre dans notre série d'un manque de spécificité (30,0\%). Une corrélation positive a été mise en évidence entre le taux HDL-C et l'hémoglobine $(r=-0,37 ; p<0,01)$ mais pas avec la densité parasitaire.

Tous les malades ont des taux de LDL-C dans la fourchette des valeurs normales $(\leqslant 130 \mathrm{mg} / \mathrm{dl}$ ) et seulement 10,0 \% des enfants du groupe contrôle ont des valeurs augmentées. Les taux de LDL-C ne sont corrélés ni à l'hémoglobine ni à la parasitémie.

\section{DISCUSSION}

P our le dosage de CT, la conservation à des températures en dessous de $-18{ }^{\circ} \mathrm{C}$ est recommandée pour les échantillons devant être conservés longtemps avant analyse par la méthode enzymatique (Allain et al., 1974; Ektachem Clinical Chemistry Slides-Chol, 1992). Le fait que le dosage ait été pratiqué sur du plasma après décongélation ne doit donc pas altérer les résultats.

Les taux de CT sont bas dans notre série, mais ils sont encore abaissés chez les malades et ce, quelque soit la gravité de la maladie. La baisse des taux de CT au

\begin{tabular}{|c|c|c|c|c|c|c|}
\hline \multirow{2}{*}{$\begin{array}{c}\text { Paramètre } \\
\text { (valeur de référence) }\end{array}$} & \multirow{2}{*}{$\begin{array}{c}\text { Contrôle (C) } \\
(n=30)\end{array}$} & \multirow{2}{*}{$\begin{array}{c}\text { Accès } \\
\text { simple (AS) } \\
(n=44)\end{array}$} & \multirow{2}{*}{$\begin{array}{c}\text { Accès } \\
\text { pernicieux (AP) } \\
(n=12)\end{array}$} & \multicolumn{3}{|c|}{ Valeur $\boldsymbol{p}$} \\
\hline & & & & C-AS & $\mathrm{C}-\mathrm{AP}$ & $A S-A P$ \\
\hline $\begin{array}{l}\text { Parasitémie } \\
(n \text { parasites/ } \mu l)\end{array}$ & - & $\begin{array}{c}34720 \\
(26100-42840)\end{array}$ & $\begin{array}{c}62100 \\
(53 \quad 480-103208)\end{array}$ & - & - & $<0,001$ \\
\hline $\begin{array}{l}\text { Hémoglobine } \\
(13,0 \pm 1,5 \mathrm{~g} / \mathrm{dl})\end{array}$ & $\begin{array}{c}9,4 \\
(8,3-11,2)\end{array}$ & $\begin{array}{c}7,8 \\
(6,9-9,9)\end{array}$ & $\begin{array}{c}5,6 \\
(5,1-6,9)\end{array}$ & $<0,05$ & $<0,001$ & $<0,001$ \\
\hline $\begin{array}{l}\text { Cholestérol } \\
(112-248 \mathrm{mg} / \mathrm{dl})\end{array}$ & $\begin{array}{c}124,5 \\
(108,0-146,0)\end{array}$ & $\begin{array}{c}90,5 \\
(72,0-115,0)\end{array}$ & $\begin{array}{c}108,5 \\
(82,0-129,5)\end{array}$ & $<0,001$ & NS & NS \\
\hline $\begin{array}{l}\text { HDL-cholestérol } \\
(36-84 \mathrm{mg} / \mathrm{dl})\end{array}$ & $\begin{array}{c}32,5 \\
(27,0-39,0)\end{array}$ & $\begin{array}{c}1,0 \\
(0,0-10,0)\end{array}$ & $\begin{array}{c}0,0 \\
(0,0-14,5)\end{array}$ & $<0,001$ & $<0,001$ & NS \\
\hline $\begin{array}{l}\text { Triglycérides } \\
(24-140 \mathrm{mg} / \mathrm{dl})\end{array}$ & $\begin{array}{c}75,0 \\
(59,0-91,0)\end{array}$ & $\begin{array}{c}135,0 \\
(91,0-172,5)\end{array}$ & $\begin{array}{c}144,5 \\
(89,5-289,0)\end{array}$ & $<0,001$ & $<0,001$ & NS \\
\hline $\begin{array}{l}\text { LDL-cholestérol } \\
(\leq 130 \mathrm{mg} / \mathrm{dl})\end{array}$ & $\begin{array}{c}76,9 \\
(61,8-103,4)\end{array}$ & $\begin{array}{c}55,7 \\
(38,9-73,6)\end{array}$ & $\begin{array}{c}57,0 \\
(35,1-80,9)\end{array}$ & $<0,01$ & NS & NS \\
\hline
\end{tabular}

Tableau I. - Paramètres parasitaires, hématologiques et lipidiques dans les différents groupes étudiés : médiane $\left(q_{1}-q_{3}\right)$. 


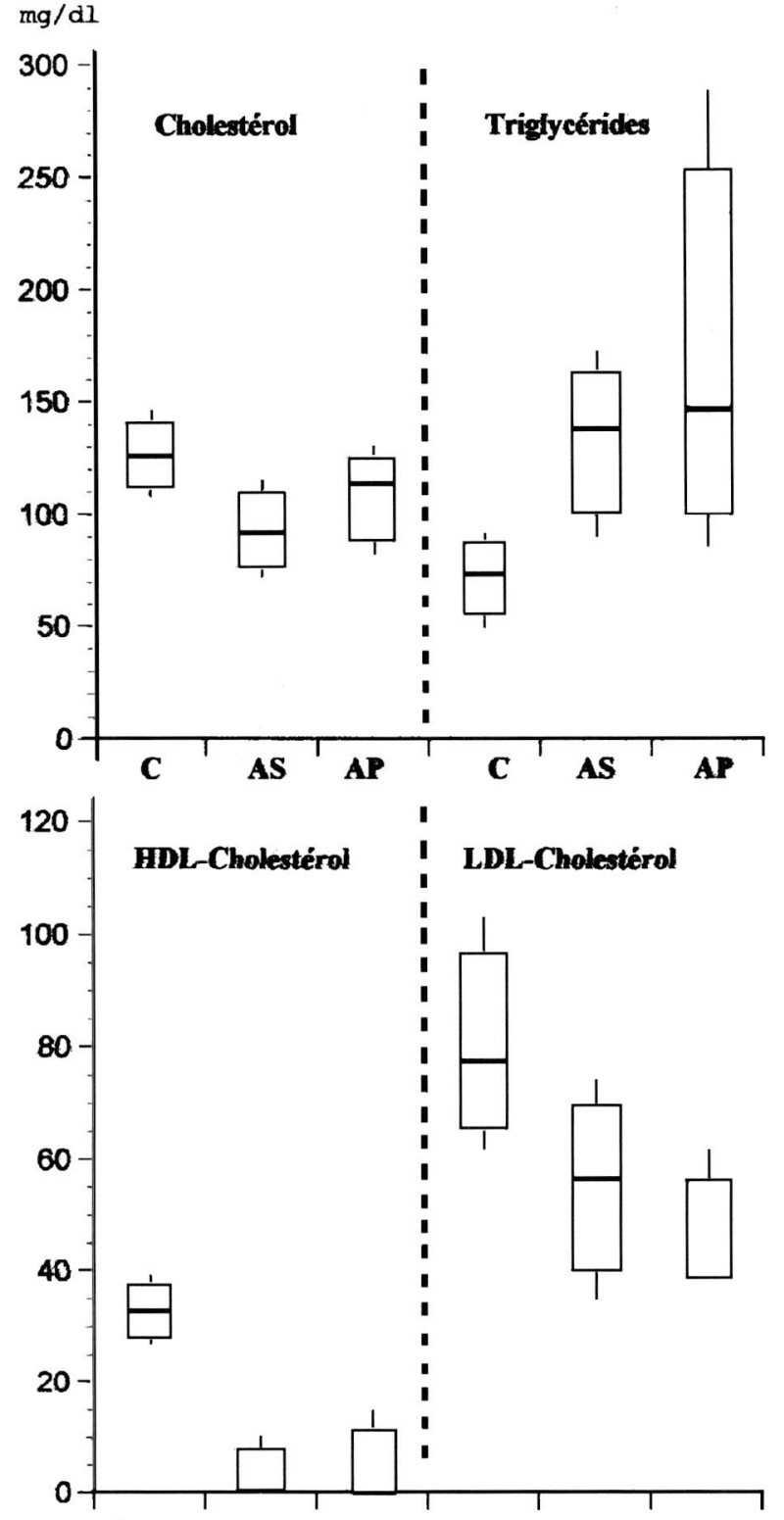

Fig. 1. - Taux lipidiques des enfants asymptomatiques (C) et des enfants infectés par Plasmodium falciparum (AS, AP). La médiane ainsi que les percentiles 25 et 75 sont représentés par les boîtes, les percentiles 10 et 90 par les lignes verticales. AS : accès simple; AP : accès pernicieux.

cours de l'accès palustre à $P$. falciparum ont également été observés par Djoumessi (1989), par Mohanty et al. (1992) et par Das et al. (1993). La différence entre accès simple et accès pernicieux est parfois signalée (Mohanty et al., 1992; Das et al., 1993). Les modifications observées sont cependant toujours modérées, parfois nulles comme chez Onongbu et Onyeneke (1983).

Dans notre série de malades, quelque soit le degré de gravité de la maladie, il y a une augmentation nette des TG. Sur ce point, tous les auteurs sont d'accord (Onongbu \& Onyeneke, 1983; Mohanty et al., 1992;
Das et al., 1993). Ce dernier auteur a même trouvé une corrélation entre TG, densité parasitaire et gravité du paludisme, fait que nous n'avons pas pu mettre en évidence, pas plus que Vernes et al. (1980). Les taux de TG sont en relation inverse avec le taux d'hémoglobine $(r=-0,37 ; p<0,05)$, comme Das et al. (1993) l'ont également observé. Dans les modèles animaux, Maurois et al. (1980) rapporte dans les infections à $P$. yoelii et $P$. chabaudi une augmentation des triglycérides proportionnelle à la densité parasitaire.

Nous avons trouvé des différences significatives des taux d'HDL-C $(p<0,001)$ entre non-malades et malades, mais sans signification avec la sévérité de la maladie. Les taux des HDL-C sont peu élévés et subissent un effondrement considérable chez nos malades. L'observation est faite par nombre d'auteurs (Djoumessi, 1989; Agbedana et al., 1990; Mohanty et al., 1992; Das et al., 1993). Nilsson-Ehle \& NilssonEhle (1990) on même suggéré que cette réduction pouvait être utilisée dans le diagnostic du paludisme, en lui attribuant une haute sensibilité. Mais on ne peut pas considérer les dosages des HDL-C comme un test " diagnostique ", à cause du manque de spécificité qui n'est, dans notre série, que de 30,0\%. Toutefois, chez les malades non immuns avec parasitémie périphérique peu élevée ou même subpatente, un taux d'HDL-C très bas augmente le degré de suspicion. A l'inverse, les taux élevés d'HDL-C peuvent jouer un rôle dans le cas d'exclusion d'un accès palustre à $P$. falciparum.

Nos résultats ne confirment pas, pour les HDL-C et dans le cadre du paludisme à $P$. falciparum, les observations de Mohanty et al. (1992), qui ont reconnu des corrélations positives entre le paludisme simple et sévère, allant jusqu'à proposer les HDL-C comme indicateur de la sévérité de la maladie. Bien que la corrélation entre le taux d'HDL-C et la sévérité de la maladie ne soit pas significative, le faible nombre de cas d'accès pernicieux de notre échantillon $(n=12)$, renvoie à de futures observations la conclusion sur l'association positive avec la sévérité de la maladie.

Le calcul de la concentration en LDL-C par la formule de Friedewald et al. (1972) est réputé fiable pour les valeurs de TG ne dépassant pas $400 \mathrm{mg} / 10 \mathrm{dl}$. Les valeurs retrouvées dans le présent travail sont toutes inférieures à la concentration limite.

Les taux des LDL-C dans notre échantillon diminuent avec la maladie (fig. 1). Mohanty et al. (1992) font référence à une corrélation positive entre les LDL-C de malades et non-malades et la sévérité de la maladie. Nos résultats peuvent seulement corroborer une corrélation significative entre l'accès simple et le groupe contrôle mais non avec la sévérité de la maladie (tableau I). 
Les mécanismes de la dyslipidémie chez l'accès palustre restent discutés et plusieurs hypothèses ont été proposées :

- Pendant l'infection à $P$. falciparum, la synthèse de lipides et phospholipides est diminuée (Davis et al., 1993a) et en même temps il y a une consommation accrue de CT et phospholipides par le parasite ou par l'érythrocyte infecté (Rao et al., 1969; Lambrecht et al., 1978).

- La diminution du CT total peut être due à la diminution des HDL-C, qui assurent leur transport vers le foie. Ce mécanisme enzymatique fait appel à la production par le parasite d'un enzyme capable d'inhiber ou de détériorer la lécithine-cholestérol-acyltransférase plasmatique (LCAT), enzyme générateur des niveaux des HDL-C (Vernes et al., 1980; Maurois et al., 1985; Djoumessi, 1989).

- Le niveau élevé des TG dans le plasma est le résultat d'une activité réduite de la lipoprotéine lipase, par défaut de l'épuration des TG du compartiment plasmatique (Maurois et al., 1980; Nilson-Ehle et al., 1980; Al-Rifai et al., 1982). L'hypothèse est basée sur la capacité d'inhibition de la lipoprotéine lipase par le Tumor Necrosis Factor, augmentée pendant l'accès palustre (Scuderi et al., 1986; Beutler \& Cérami, 1987; Semb et al., 1987; Grau et al., 1989).

- La corrélation positive entre les taux d'albumine (protéine de phase aiguë qui baisse aussi dans l'accès palustre) et les taux des HDL-C pourrait être la conséquence de la réponse de phase aiguë à l'infection (Mohanty et al., 1992). La baisse de l'albumine serait due à une fuite des protéines plasmatiques suite à l'augmentation de la perméabilité transcapillaire ou à une diminution de la synthèse (à cause de l'activité réduite de la lipoprotéine lipase).

Qu'elles soient sous la dépendance du métabolisme lipidique du parasite ou des relations hôte-parasite, le rôle des modifications du métabolisme des lipides de l'érythrocyte parasité reste à préciser. Une meilleure connaissance des mécanismes par lesquelles le parasite récolte les lipides (ou leurs précurseurs) indispensables à sa survie, devrait permettre de trouver de nouveaux médicaments capables de déstabiliser le métabolisme lipidique du parasite (Slater \& Cerami, 1992; Grellier et al., 1994) et d'expliquer certains mécanismes de la résistance parasitaire croissante à la chloroquine et à la quinine des souches de P. falciparum (Sherwood et al., 1986; Vial et al., 1992).

\section{REMERCIEMENTS}

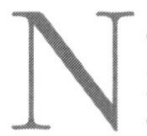

ous tenons à remercier l'infirmier de santé publique, Mário Lopes Gomes, et le médecin chef de la mission française de coopération pour la santé à São Tomé et Principe, Dr. Pierre Cochet, pour leur aide dans la réalisation de l'enquête.

\section{RÉFÉRENCES}

Agbedana E.O., Salimonu L.S., Taylor G.O. \& Williams A.I.O. Studies of total and high density lipoprotein cholesterol in childhood malaria: a preliminary study. Annals of Tropical Medicine and Parasitology, 1990, 84, 529-530.

Al-Rifai W., Maurois P., Fruchart J.C. \& Charet P. Adipose tissue lipoprotein lipase activity in Plasmodium chabaudi and Plasmodium vinckei rodent malaria. Biochimie, 1982 , 64, 127-132.

Allain C.C., Poon L.S., Chan C.S.G., Richmond W. \& Fu P.C. Enzymatic determination of total serum cholesterol. Clinical Chemistry, 1974, 20, 470-475.

Angus M.G.N., Fletcher K.A. \& Maegraith H.B.G. Studies on the lipids of Plasmodium knowlesi-infected rhesus monkeys (Macaca mulatta). I. Changes in serum lipids: Annals of Tropical Medicine and Parasitology, 1971, 65, 135-154.

BARkin R.M. \& Rosen P. Emergency Pediatrics. A guide to ambulatory care (3e edition) 1990, The CV Mosby Company (eds), Los Angeles.

Beutler B. \& Cerami A. Cachectin: more than a tumor necrosis factor. The New England Journal of Medicine, 1987, 316, 379-385.

Borvin P. Membrane érythrocytaire ( $2^{\mathrm{e}}$ édition). In: Hématologie. Bernard D., Breton-Gorius J., Rochant H., Reyes F., Vernant J.P. (eds), Flammarion Médecine-Sciences, Paris, 1986, 76-77.

Bruce-Chwatt L.J., Black R.H., Canfield C.J., Clyde D.F., Peters W. \& Wernsdorfer W.H. Chimiothérapie du paludisme ( $2^{\mathrm{e}}$ édition), OMS, Genève, 1984, 207-212.

Cuisinier-Rayal J.C., Bire F., Clerc M., Bernard J. \& Sarrouy J. Le syndrome dysglobulinémie-hypocholestérolémie. Médecine Tropicale, 1990, 50, 91-95.

Das B.S., Patnaik J.K., Mohanty S., Mishra S.K., Mohanty D., SAtPathy S.K \& Bose T.K. Plasma antioxidants and lipid peroxidation products in falciparum malaria. American Journal of Tropical Medicine and Hygiene, 1993, 49, 720725 .

Davis T.M., Sturm M., Zhang Y.R., Spencer J.L \& Granham R.M., GUO-QiaO L. \& TAYlor R.R. Platelet-activating factor and lipid metabolisme in acute malaria. Journal of Infection, 1993a, 26, 279-285

Davis T.M.E., Brown A.E. \& SMITH C.D. Metabolic disturbances in Plasmodium coatneyi-infected rhesus monkeys. International Journal for Parasitology, 1993, 23, 557-568.

Djoumessi S. Serum lipids and lipoproteines during malaria infection. Pathologie Biologie, 1989, 37, 909-911.

Ektachem clinical chemistry slides (chol), Johnson EJohnson Clinical Diagnosis, MP2-35, CAT n 8092959, 1992, 1-5.

FRIEDEWALD W.T., LEVY R.I \& FREDRICKSON D.S. Estimation of the concentration of low-density lipoprotein cholesterol in plasma, without use of the preparative ultracentrifuge. Clinical Chemistry, 1972, 18, 499-502.

Grau G.E., Taylor T.E., Molineaux M.E., Wirima J.J., Vassali P., Hommel M \& LamberT P.H. Tumor necrosis factor and disease severity in children with falciparum malaria. The New England Journal of Medicine, 1989, 320, 1586-1591. 
Greller P., Rigomier D., Clavey V., Fruchart J.C. \& Schrevel J. Lipid traffic between high density lipoproteins and Plasmodium falciparum-infected red blood cells. Journal of Cell Biology, 1991, 112, 267-277.

Grellier P., Valentin A., Millerioux V., Schrevel J. \& RigoMIER D. 3-hydroxy-3-methylglutaryl coenzyme A reductase inhibitors lovastatin and simvastatin inhibit in vitro development of Plasmodium falciparum and Babesia divergens in human erythrocytes. Antimicrobial Agents and Chemotherapy, 1994, 38, 1144-1148.

Hartmann L. \& Schneider J. Étude des lipoprotéines et de la Bêta-2 macroglobuline des paludéens. Complément de recherches consacrées aux exploitations fonctionnelles hépatiques au cours du paludisme. Annales de la Société Belge Médecine Tropicale, 1963, 4, 503-510.

Holz Jr. G.G. Lipids and the malarial parasites. Bulletin of the World Health Organization, 1977, 55, 237-248.

Lambrecht A.J., Snoeck J. \& Timmermans U. Transient an-alphalipoproteinaemia in man during infection by Plasmodium vivax. The Lancet, 1978, $i, 1206$.

LÉvy-Lambert E. Techniques de base pour le laboratoire médical, OMS/Genève, 1973, 251-253.

Maguire P.A. \& Sherman I.W. Phospholipidid composition, cholesterol content and cholesterol exchange in Plasmodium falciparum-infected red cells. Molecular and Biochemical Parasitology, 1990, 38, 105-112.

Maurois P., Charet P., Nouvelot A., Fruchart J.C., Vernes A. \& Biguet J. Kinetic study of serum lipoproteines, total cholesterol and triacylglycerides in various models of experimental rodent malaria. Annals of Tropical Medicine and Parasitology, 1980, 74, 17-28.

Maurois P., Pessah M., Bricge I. \& Alcindor L.G. Alterations of lecithin-cholesterol-acyltransferase activity during Plasmodium chabaudi rodent malaria. Biochimie, 1985, 67, 227-239.

Maurois P., Vernes A. \& Charet P. Changes in serum lipoproteins during malaria therapy with Plasmodium vivax. Annals of Tropical Medicine and Parasitology, 1979, 5, 491493.

Mohanty S., Mishra S.K., Das B.S., Satpathy S.K., Mohanty D., Patnaik J.K. \& Bose T.K. Altered plasma lipid pattern in falciparum malaria. Annals of Tropical Medicine and Parasitology, 1992, 86, 601-606.

Muller C.P. \& Shinitzky M. Passive shedding of erythrocyte antigens induced by membrane rigidification. Experimental Cell Research, 1981, 136, 53-62.

Nilsson-Ehle I. \& Nilsson-Ehle P. Changes in plasma lipoproteines in acute malaria. Journal of Internal Medicine, 1990, 277, 151-155.

Nilsson-Ehle P., Garfinkel A.S. \& Schotz M.C. Lipolytic enzymes and plasma lipoprotein metabolism. Annual Review of Biochemistry, 1980, 49, 667-693.

Onongbu I.C. \& Onyeneke E.C. Plasma lipid changes in human malaria. Tropenmedizin und Parasitologie, 1983, 34, 193-196.

RaO K.N., Subrahmanyam D. \& Prakash S. Studies on the lipides of plasma of rats infected with Plasmodium ber- ghei. Indian Journal of Medical Research, 1969, 57, 921924.

RIFAI N. \& WARNICK G.R. Methods for clinical laboratory measurement of lipid and lipoprotein risk factors. American Association for Clinical Chemistry (eds), AACC Press,. Washington DC, 1991.

SAmuel Meites. Pediatric Clinical Chemistry: reference (normal) values ( $3^{\mathrm{e}}$ ed.), AACC Press (eds), Washington DC, 1988.

Sandstad J.S., Mckenna R.W. \& Keffer J.H. Handbook of clinical pathology, American Society of Clinical Pathologists (eds), Chicago, 1992, 77-80.

Scuderi P., Sterling K.E., Lam K.S., Finley P.R., Ryan K.J., Ray C.G., Peterson E., Slymen D.J. \& Salmon S.E. Raised serum levels of tumor necrosis factor in parasitic infection. The Lancet, 1986, ii, 1364-1365.

Semb H., Peterson J., Tavernier J. \& Olivecrona T. Multiple effects of tumor necrosis factor on lipoproteine lipase. Journal of Biological Chemistry, 1987, 262, 8390-8394.

Sherwood J.A., Spitalnik S.L., Aley S.B., Quakyi I.A. \& Howard R.J. Plasmodium falciparum and Plasmodium knowlesi: Initial Identification and Characterization of Malaria Synthesized Glycolipids. Experimental Parasitology, 1986, 62, 127-141

Slater F.G. \& Cerami A. Inhibition by chloroquine of a novel haem polymerase enzyme activity in malaria trophozoites. Nature, 1992, 355, 167-169.

Spayd R.W., Bruschi B., Burdick B.A., Dappen G.M., Eikenberry J.N., Esders W., Figueras J., Goodhue C.T., Larossa D.D., Nelson R.W., Rand R.N. \& Wu T.W. Multilayer film elements for clinical analysis: applications to representative chemical determinations. Clinical Chemistry, 1978, 8, 1343-1350

Vernes A., Dei-Cas E., Dutoit E., Maurois P. \& Gentilini J.L., Biguet J. Modifications du lipoprotéinogramme au cours du paludisme humain. Pathologie Biologie, 1980, 28, $457-$ 460.

Vial H. J., Angelin M.L., Elabbadi N., Calas M., Cordinas G. \& GIRAL L. Basic biochemical investigations as rationale for the design of original antimalarial drugs. An example of phospholipid metabolisme. Memórías do Instituto Oswaldo Cruz, 1992, 87 (suppl. 3), 251-261.

Warrel D.A., Molineaux L. \& Beales P.F. Severe and complicated malaria. ( $2^{\mathrm{e}}$ edition). Transactions of the Royal Society of Tropical Medicine and Hygiene (suppl. 2), 1990, $84,1-59$

Wéry M. Diagnostic biologique. In: Paludisme. Danis M. Mouchet J., Ellipses/Aupelf (eds), Paris, 1991, 114-117.

Wunderlich F., Fiebig S., Vial H. \& Kleinig H. Distint lipid compositions of parasite and host cell plasma membranes from Plasmodium chabaudi-infected erythrocytes. Molecular and Biochemical Parasitology, 1991, 44, 271-278. 